\title{
Review: telephone and computer communication improves healthcare process and patient outcomes
}

\author{
Balas EA, Jaffrey F, Kuperman GJ, et al. Electronic communication with patients. Evaluation of distance medicine technology.JAMA \\ 1997 Jul 9;278:152-9.
}

\section{Objective}

To determine the efficacy of distance healthcare technologies for clinical process and patient outcomes.

\section{Data sources}

Randomised controlled trials (RCTs) were identified from 1966-96 in the Columbia Registry of Information and Utilization Management Trials and other databases (Medline, HSTAR, PsychLIT, CINAHL, Science Citation Index, and Engineering Index) using the search terms telephone and computer.

\section{Study selection}

Studies were included if they evaluated electronic communication (computer or telephone) between people in separate locations, included a control group that did not have a similar intervention, and evaluated process or patient care outcomes.

Efficacy of distance healthcare technologies

\section{Data extraction}

Data were extracted on description and quantification of study quality, intervention, intervention site, providers, patients, and care process or patient outcomes.

\section{Main results}

See table.

\section{Conclusion}

Distance healthcare technologies (electronic communication) can improve clinical process and patient care outcomes.

Sources of funding: National Library of Medicine; National Science Foundation; Arizona State University.

For article reprint:Dr E A Balas, School of Medicine, University of Missouri-Columbia, 324 Clark Hall, Columbia, MO 65211, USA. Fax +1 5738826158

Adapted from an abstract published in ACP Journal Club 1998 Jan-Feb;128:22.
Balas $e t$ al have produced a thorough systematic review on process and outcome studies evaluating use of telehealth. Studies were limited to trials examining the use of telephones and computers to facilitate the delivery of health care. Most of the advanced and more recent types of telehealth use (eg, live audio visual consultations) have been evaluated by descriptive methods, rather than analytical designs. ${ }^{1}$ Using standardised methods, high quality RCTs were selected from a range of specialties. The results suggest benefits for the specific populations studied, including people with osteoarthritis, cardiac events, or diabetes. 26 trials used nurse initiated contact; however, it was not clear which studies used this approach and whether they were effective.
Several of the studies report cointervention bias (clinic visits were offered in addition to telephone support).

This review provides valuable information on the benefits for patients with access to telehealth delivery. The information is relevant to nurses who provide telephone advice and counselling to clients in the community. For example, as health promotion and disease prevention initiatives expand at the community level, nurses will play a fundamental part in teaching patients and families how to manage conditions and alert them to the need for a physician visit. ${ }^{2}$ Evidence that after hours telephone services for chronically ill patients can reduce hospital use and increase caregiver satisfaction is beneficial for nurses who provide these types of services and who plan to evaluate their own service. Positive outcomes for the studies of computerised communication will be helpful to nurses who may participate in more advanced telehealth services such as telemonitoring of patients in the home.

Janet Yamada, RN, BScN Research Coordinator/Staff Nurse

The Ontario Regional Poison Control Centre The Hospital for Sick Children Toronto, Ontario, Canada

1 Perednia DA, Allen A. JAMA 1995;273:483-8. 2 Rachlis M, Kushner C. Strong medicine: how to save Canada's health care system. Toronto: HarperCollins; 1994. 